\title{
Estimação de parâmetros genéticos para características de pesos e pesos metabólicos na desmama e pós-desmama em bovinos Brahman
}

\author{
[Estimation of genetic parameters for weaning weights and metabolic weights characteristics and post \\ weaning in Brahman cattle]
}

\author{
M. Manuel, L. Cavani, T.J. Menezes, D.D. Millen, C. Andrighetto, G.C. Lupatini, R. Fonseca
}

Universidade Estadual Paulista Júlio de Mesquita Filho - Dracena, SP

\section{RESUMO}

Objetivou-se estimar herdabilidades e correlações de características ponderais com 36.505 animais, da Associação Brasileira de Criadores Zebu. O modelo incluiu efeito genético direto, materno, ambiente permanente, residual - aleatórios e efeitos de grupos contemporâneos - fixos. Os parâmetros foram estimados pelo método de máxima verossimilhança restrita (REML), utilizando-se software Wombat. Os resultados das herdabilidades variaram de 0,20 a 0,25 peso à desmama e ao sobreano; 0,16 a 0,20 peso metabólico não ajustado e ajustado à desmama, 0,21 a 0,25 peso metabólico ajustado à desmama e metabólico ajustado ao sobreano. As correlações genéticas entre peso à desmama e peso metabólico não ajustado à desmama, peso à desmama e peso metabólico ajustado à desmama são, respectivamente $0,76 \mathrm{e}$ 1,00. A correlação genética entre peso ao sobreano e metabólico ao sobreano não ajustado, peso ao sobreano com metabólico sobreano ajustado foram 0,97 e 1,00 . Correlação genética entre peso à desmama e ao sobreano foi 0,72 , peso metabólico não ajustado à desmama e metabólico não ajustado ao sobreano 0,54, peso metabólico ajustado à desmama e metabólico ajustado ao sobreano foi 0,71 . Correlações genéticas entre peso à desmama e metabólico ajustado à desmama e peso ano com metabólico ano ajustado foram 1,00 e 1,00. Portanto, utilização de peso metabólico sem ajuste de idade pode viesar estimativas de parâmetros genéticos.

Palavras-chave: componentes de variância, correlação genética, herdabilidade

\begin{abstract}
The objective of this study was to estimate heritability and correlations of 36,505 animals, belonging to the Brazilian Association of Zebu Breeders. They were estimated by Restricted Maximum Likelihood method (REML) using Wombat software. The model included the direct additive genetic effect, maternal, permanent maternal environment and residual as random and fixed effects of contemporary group. The results of heritability ranged from 0,20 to 0,25 for weaning weight and yearling; 0,16 to 0,20 for real metabolic weaning, 0,21 to 0,25 for metabolic adjusted weight at weaning and yearling metabolic adjusted. The genetic correlations between weaning weight with metabolic real, weaning weight adjusted with metabolic are respectively 0,76 and 1,00. The genetic correlation between yearling weight and metabolic real, yearling weight adjusted metabolic were 0,97 and 1,00. Genetic correlations between weaning weight and yearling was 0,72 , real metabolic weight at weaning and yearling real metabolic was 0,54, adjusted metabolic weight at weaning and yearling metabolic adjusted was 0,71. Genetic correlations between weight at weaning and adjusted metabolic and weight-adjusted metabolic year were 1,00 and 1,00. Therefore, the use of metabolic weight without age adjustment can warn the estimates.
\end{abstract}

Keywords: variance components, genetic correlation, heritability

Recebido em 8 de fevereiro de 2017

Aceito em 5 de julho de 2017

E-mail: manuelsoaresvet@gmail.com 


\section{INTRODUÇÃO}

As principais fontes de informações das avaliações genéticas de gado de corte são os pesos observados ao longo da vida dos animais, por serem de fácil mensuração e interpretação, além de, geralmente, apresentarem herdabilidade de magnitudes médias a altas, indicativo de que a seleção poderá resultar em progresso genético.

Com vista em melhorar as acurácias de seleção, os programas de melhoramento genético estabelecem fatores de ajuste matemático ou procedimentos de ajuste dos pesos para determinadas idades, os quais passam a ser chamados de pesos ajustados. $O$ peso ao desmame em bovinos de corte fornece um exemplo típico de um procedimento de ajuste matemático para explicar os efeitos ambientais identificáveis (como o da idade). Os pesos ao desmame são normalmente ajustados para a idade do bezerro e a idade da mãe do bezerro. Essas idades recebem denominação de idadespadrão e são adotadas segundo princípios biológicos ou meramente cronológicos (Sakaguti et al., 2003). Entretanto, a seleção para pesos ajustados tende a tornar o sistema de produção mais oneroso, uma vez que animais maiores tendem a comer maior quantidade de alimento, aumentando os custos com a alimentação do rebanho.

Para contribuir com a diminuição de custos na alimentação, buscam-se fêmeas (por serem a categoria mais numerosa em um rebanho de ciclo completo) de tamanho médio, uma vez que essas consumirão menos alimento e tenderão a pesar menos e, consequentemente, não apresentarão problemas com partos distócicos. O ideal é que se tenham matrizes que demandam menor energia de manutenção para um determinado peso adulto, ou seja, buscam-se matrizes de menor peso metabólico. Embora grande ênfase seja dada às matrizes, essa característica também é importante para todas as outras categorias de animais dentro da propriedade, uma vez que animais com menor demanda de energia de manutenção devem comer menos e contribuir para a diminuição de custos em quase todas as raças.

De acordo com Heady (1975), o peso metabólico proporciona o melhor meio de comparação entre animais com idades diferentes, visto que animais menores produzem mais calor e consomem mais alimento por unidade de tamanho corporal do que animais maiores, pois a taxa metabólica basal varia.

As necessidades energéticas de um animal dependem mais do seu peso metabólico do que do seu peso vivo. Assim, seria possível pensar no peso metabólico como um critério de seleção e não simplesmente como um componente constituinte de outra característica. Dada a afirmação de Heady (1975), seria possível utilizar o peso metabólico como componente de uma característica sem qualquer ajuste para idade. Entretanto, a utilização do peso metabólico como critério de seleção demanda ajuste prévio para a idade, e, nesse caso, o peso metabólico e o peso à idade-padrão correspondente são medidas proporcionais que possuem estrutura de variância e covariância equivalentes e produzirão os mesmos valores de herdabilidade e correlações genéticas. Portanto, utilizar pesos metabólicos ajustados e pesos à idade-padrão tem o mesmo efeito sob o ponto de vista da estimação de parâmetros genéticos e da predição de valores genéticos aditivos, e não deve contribuir para o progresso genético da população de animais com relação ao consumo de menor quantidade de energia de manutenção.

A utilização de pesos metabólicos não ajustados para as idades, entretanto, pode gerar erros de grandes magnitudes, que podem influenciar de forma determinante a tomada de decisões estratégicas e a seleção de reprodutores como futuros pais dentro de um programa de melhoramento. Dessa forma, o objetivo deste estudo foi demonstrar as consequências do emprego do peso metabólico, sem qualquer ajuste para a idade, sobre a estimação de componentes de variância e predição de valores genéticos.

\section{MATERIAL E MÉTODOS}

O experimento foi realizado a partir de registros de animais em arquivo de dados de pesos com 36.505 bovinos da raça Brahman do programa de Associação Brasileira dos Criadores de Zebu (Associação Brasileira dos Criadores de Zebu, ABCZ), provenientes dos estados de São Paulo, Minas Gerais, Rio de Janeiro, Espírito Santo, Distrito Federal, Mato Grosso, Mato Grosso do Sul e Goiás, nascidos entre os anos de 1996 a 
2012. Para este estudo, foram extraídos do banco de dados animais exclusivamente criados a pasto.

As idades-padrão foram calculadas com base nos procedimentos de ajustamento dos programas de melhoramento genético de raças zebuínas existentes no Brasil. Para que o animal tenha seu peso calculado a idades-padrão de 205, 365 ou 550 dias, foi necessário que pelo menos uma das pesagens consideradas estivesse dentro das faixas de mais ou menos 50 dias: 205 dias (155 a 255 dias); 365 dias (315 a 415 dias); 550 dias (500 a 600 dias). Os animais que não atenderam a esse requisit, ficaram sem o cálculo naquela(s) idade(s)-padrão. Os cálculos de pesos à idadepadrão foram feitos de acordo com a fórmula seguinte:

$$
\mathrm{PA}=(\mathrm{Pf}-\mathrm{Pi} / \mathrm{D}) * \mathrm{C}+\mathrm{Pi},
$$

em que: $P A$ é o peso à idade-padrão $(\mathrm{kg}), P f$ peso final $(\mathrm{kg}), P i$ peso inicial $(\mathrm{kg}), D$ número de dias entre pesagens e $C$ ajuste em dias. $\mathrm{O}$ peso metabólico ajustado foi calculado com base no peso á idade-padrão elevado a $0,75 \mathrm{PA}^{0,75}$, e o peso metabólico não ajustado foi calculado com base no peso bruto elevado a $0,75 \mathrm{P}^{0,75}$.

O banco de dados foi submetido à análise de consistência e, posteriormente, a um estudo sobre os efeitos ambientais para a formação dos grupos contemporâneos (GC). As duas etapas foram realizadas utilizando-se o software " $R$ " (R Development Core Team, 2013).

$\mathrm{O}$ arquivo de dados foi montado criando-se as seguintes características: pesos ajustados a idades-padrão de 205 (PA205), 365 (PA365) e 550 (PA550) dias, pesos metabólicos ajustados à idade-padrão de $205\left(\mathrm{PA}^{0,75} 205\right), 365\left(\mathrm{PA}^{0,75} 365\right)$ e $550\left(\mathrm{PA}^{0,75} 550\right)$ dias, pesos metabólicos não ajustados de $205\left(\mathrm{P}^{0,75} 205\right), 365\left(\mathrm{P}^{0,75} 365\right)$ e 550 $\left(\mathrm{P}^{0,75} 550\right)$ dias.

Durante a consistência dos dados, foram retirados do arquivo animais com pai e mãe desconhecidos, animais com pesos ao nascer menores que $15 \mathrm{~kg}$ ou maiores que $50 \mathrm{~kg}$ e grupos de contemporâneos constituídos com menos de três animais.

Os efeitos ambientais que influenciaram cada característica foram determinados com auxílio do método dos quadrados mínimos, utilizando-se o software "R" (R Development Core Team, 2013).
A formação de grupo de contemporâneos (GC) foi definida pelas variáveis: criador e fazenda de origem do animal, sexo, estação de nascimento e o ano de nascimento. Um total de 1659 níveis de GC foi utilizado nas análises. Após a consistência do arquivo de dados, as análises bicaracterísticas foram realizadas com um total de 47.781 animais, filhos de 2.984 touros e 17.930 vacas.

Para estimar os parâmetros genéticos, análises bicaracterísticas foram realizadas empregando-se a metodologia da máxima verossimilhança restrita (REML) por meio do software Wombat (Meyer, 2011). Em todas as análises, foi considerado o modelo animal. O critério de convergência para todas as análises foi $10^{-9}$.

Foram utilizados dois modelos. O modelo estatístico (M1) incluiu o efeito genético aditivo direto, como aleatório, além dos efeitos fixos de GC:

$y=X \beta+Z a+e$.

Esse modelo foi utilizado para a análise das características pesos ajustados às idades-padrão de 365 (PA365), 550 (PA550) dias, pesos metabólicos ajustados às idades-padrão de 365 $\left(\mathrm{PA}^{0,75} 365\right)$ e $550 \quad\left(\mathrm{PA}^{0,75} 550\right)$ dias, pesos metabólicos não ajustados às idades de 365 $\left(\mathrm{P}^{0,75} 365\right)$ e $550\left(\mathrm{P}^{0,75} 550\right)$ dias.

O modelo estatístico (M2) incluiu os efeitos genéticos diretos e maternos e o efeito de ambiente materno $y=X \beta+Z a+M m+W p e+e$,

em que: $y$ é o vetor de registros para cada média de pesos, $\beta$ é o vetor de efeitos fixos (GC), $a$ é o vetor de efeito genético aditivo direto, $m$ é o vetor de efeito genético materno, $p e$ é o vetor dos efeitos ambientais maternos permanente, e $e$ é o vetor de efeito residual aleatório. $X, Z, M e W$ são as matrizes de incidência associadas com seus respectivos efeitos de $y$.

Esse modelo foi utilizado para a análise das características de pesos ajustados para idadespadrão de 205 (PA205), pesos metabólicos ajustados à idade-padrão de $205\left(\mathrm{PA}^{0,75} 205\right)$ e pesos metabólicos não ajustados à idade de 205 $\left(\mathrm{P}^{0,75} 205\right)$. Os pressupostos para o modelo geral foram como se segue: 
$\mathrm{E}(\mathrm{y})=\mathrm{X} \beta$

$\mathrm{E}(a)=0, \mathrm{E}(m)=0, \mathrm{E}(p e)=0$ e $\mathrm{E}(e)=0$.

$\sigma_{p e}^{2}=$ variância de ambiente permanente e $\sigma_{e}^{2}=$ variância residual.

Para estimação de componentes de (co)variância, análises unicaracterísticas foram inicialmente realizadas com o objetivo de se obterem valores iniciais mais adequados para os componentes de variância das análises bicaracterísticas.

Os componentes de (co)variância estimados nas análises bicaracterísticas foram utilizados para a predição dos valores genéticos aditivos.

\section{RESULTADOS E DISCUSSÃO}

em que: $\mathrm{A}=$ matriz de parentesco genético aditivo, $I_{n v}$ e $I_{n b}=$ matrizes identidade de dimensão número de mães $(n v)$ e total de observações $(n b), \sigma_{a}^{2}=$ variância genética aditiva direta, $\sigma_{m}^{2}$ variância genética aditiva materna,

Houve diminuição considerável no número de animais avaliados para as características das idades jovem à adulta (Tab. 1).

Tabela 1. Estatística descritiva para características de pesos ajustados a idades-padrão de 205 (PA205), 365 (PA365), 550 (PA550) dias, pesos metabólicos ajustados à idade-padrão de 205 ( $\left.\mathrm{PA}^{0,75} 205\right), 365$ $\left(\mathrm{PA}^{0,75} 365\right), 550\left(\mathrm{PA}^{0,75} 550\right)$ dias e pesos metabólicos não ajustados a idades-padrão de $205\left(\mathrm{P}^{0,75} 205\right)$, $365\left(\mathrm{P}^{0,75} 365\right)$ e $550\left(\mathrm{P}^{0,75} 550\right)$ dias em bovinos Brahman

\begin{tabular}{cccccccc}
\hline Características & Animais & GC & Min & Max & Média & DP & CV $(\%)$ \\
\hline PA205 & 22865 & 1659 & 129,14 & 347,39 & 198,03 & 27,46 & 13,87 \\
PA365 & 11930 & 1344 & 208,07 & 459,73 & 273,79 & 38,84 & 14,19 \\
PA550 & 14398 & 1252 & 243,1 & 578,32 & 342,2 & 54,67 & 15,98 \\
PA $^{0,75} 205$ & 22866 & 1659 & 38,31 & 80,47 & 52,7 & 5,47 & 10,38 \\
PA $^{0,75} 365$ & 11930 & 1344 & 54,78 & 99,28 & 67,19 & 7,04 & 10,48 \\
PA $^{0,75} 550$ & 14398 & 1252 & 61,57 & 117,93 & 79,38 & 9,4 & 11,84 \\
$\mathrm{P}^{0,75} 205$ & 26613 & 1832 & 43,08 & 78,83 & 53,16 & 6,32 & 11,89 \\
$\mathrm{P}^{0,75} 365$ & 16426 & 1635 & 59,25 & 93,11 & 67,33 & 6,52 & 9,68 \\
$\mathrm{P}^{0,75} 550$ & 20683 & 1616 & 64,94 & 113,26 & 78,09 & 9,43 & 12,08 \\
\hline
\end{tabular}

GC: grupos de contemporâneos, Min: valores mínimos, Max: valores máximos, DP: desvio-padrão e CV\%: coeficiente de variação.

O abate, a mortalidade ou a venda dos animais podem explicar os resultados na variação dos valores observados. Resultados semelhantes para características de peso foram obtidos em estudos anteriores em zebuínos por Guidolin et al. (2012) para pesos na desmama e na pós-desmama e por Zuin et al. (2012) para características de crescimento em bovinos da raça Nelore. O comportamento observado na variação do número de animais pode estar associado principalmente à edição de dados (por exemplo, durante a formação de GC e o ajuste dos pesos). As diferenças de médias fenotípicas observadas para características estudadas foram ligeiramente mais altas do que as obtidas por Vargas et al. (2014), que trabalharam com a raça Brahman no Brasil para características PA210, PA365 e PA550. Além disso, a média PA205 foi próxima dos valores reportados em bovinos de raça Brahman nas regiões tropicais do México, da Venezuela e da África do Sul (Pico et al., 2004; Plasse et al., 2004; Parra-bracamonte et al., 2007; Estrada-león et al., 2014). Embora os estudos citados tenham sido realizados sob condições tropicais para a mesma raça, as diferenças entre as estimativas pode ser atribuída a diferenças dos bancos de dados das populações diferentes estudadas. Para a raça Brahman, vale ressaltar que são escassas na literatura informações referentes a estimativas de parâmetros genéticos e médias para as mais diversas características de pesos metabólicos ajustados à idade-padrão de 205 ( $\left.\mathrm{PA}^{0,75} 205\right), 365$ $\left(\mathrm{PA}^{0,75} 365\right), \quad 550 \quad\left(\mathrm{PA}^{0,75} 550\right)$ dias e pesos metabólico não ajustado aos 205 ( $\left.\mathrm{P}^{0,75} 205\right), 365$ 
$\left(\mathrm{P}^{0,75} 365\right)$ e $550\left(\mathrm{P}^{0,75} 550\right)$ dias, sobretudo no Brasil, onde a raça foi introduzida recentemente.

As estimativas de herdabilidade para características de pesos metabólicos não ajustados a diferentes idades estão, de modo geral, distintas em relação àquelas para pesos ajustados a idades-padrão e pesos metabólicos ajustados à idade-padrão (Tab. 2).
A diferença nas estimativas de herdabilidades pode ser devido ao incremento proporcional no valor da variância residual dos pesos metabólicos não ajustados em relação aos ajustados a idadespadrão e pesos metabólicos ajustados à idadepadrão. Essa situação fica mais bem ilustrada na Fig. 1.

Tabela 2. Estimativas médias das variâncias genéticas aditivas direta $\left(\sigma_{\mathrm{a}}^{\wedge}\right)$ e materna $\left(\sigma_{\mathrm{m}}{ }^{2}\right)$, variância de ambiente permanente materno $\left(\sigma_{\mathrm{pe}}{ }^{2}\right)$, variância residual $\left(\sigma_{\mathrm{e}}^{\wedge}\right)$, herdabilidade direta $\left(\mathrm{h}_{\mathrm{a}}^{\wedge}\right)$, herdabilidade materna $\left({\hat{\mathrm{h}_{\mathrm{m}}}}^{2}\right)$ e herdabilidade de ambiente permanente materno $\left(\sigma_{\mathrm{pe}}{ }^{2}\right)$ em bovinos Brahman

\begin{tabular}{cccccccc}
\hline Características & $\sigma_{\mathrm{a}}{ }^{2}$ & $\sigma_{\mathrm{m}}{ }^{2}$ & $\sigma_{\mathrm{pe}}{ }^{2}$ & $\sigma_{\mathrm{e}}{ }^{2}$ & $h_{\mathrm{a}}{ }^{2}$ & $h_{\mathrm{m}}{ }^{2}$ & $h_{\mathrm{pe}}{ }^{2}$ \\
\hline PA205 & 115,44 & 29,61 & 58,42 & 361,34 & $0,20 \pm 0,02$ & $0,05 \pm 0,01$ & $0,10 \pm 0,01$ \\
PA365 & 188,56 & & & 712,47 & $0,21 \pm 0,02$ & & \\
PA550 & 383,45 & & & 1166,8 & $0,25 \pm 0,02$ & & \\
$\mathrm{PA}^{0,75} 205$ & 4,64 & 1,19 & 2,35 & 14,29 & $0,21 \pm 0,02$ & $0,05 \pm 0,01$ & $0,11 \pm 0,01$ \\
$\mathrm{PA}^{0,75} 365$ & 6,49 & & & 23,49 & $0,22 \pm 0,03$ & & \\
$\mathrm{PA}^{0,75} 550$ & 11,57 & & & 34,26 & $0,25 \pm 0,02$ & & \\
$\mathrm{P}^{0,75} 205$ & 4,52 & 0,64 & 2,11 & 21,67 & $0,16 \pm 0,02$ & $0,02 \pm 0,01$ & $0,07 \pm 0,01$ \\
$\mathrm{P}^{0,75} 365$ & 4,04 & & & 24,73 & $0,14 \pm 0,02$ & & \\
$\mathrm{P}^{0,75} 550$ & 11 & & & 43,8 & $0,20 \pm 0,02$ & & \\
\hline
\end{tabular}

Pesos ajustados a idades-padrão 205 (PA205), 365 (PA365), 550 (PA550) dias, pesos metabólicos ajustados a idadespadrão 205 ( $\left.\mathrm{PA}^{0,75} 205\right), 365$ ( $\left.\mathrm{PA}^{0,75} 365\right), 550\left(\mathrm{PA}^{0,75} 550\right)$ dias, pesos metabólicos não ajustados a idades-padrão 205 $\left(\mathrm{P}^{0,75} 205\right), 365\left(\mathrm{P}^{0,75} 365\right)$ e $550\left(\mathrm{P}^{0,75} 550\right)$ dias.

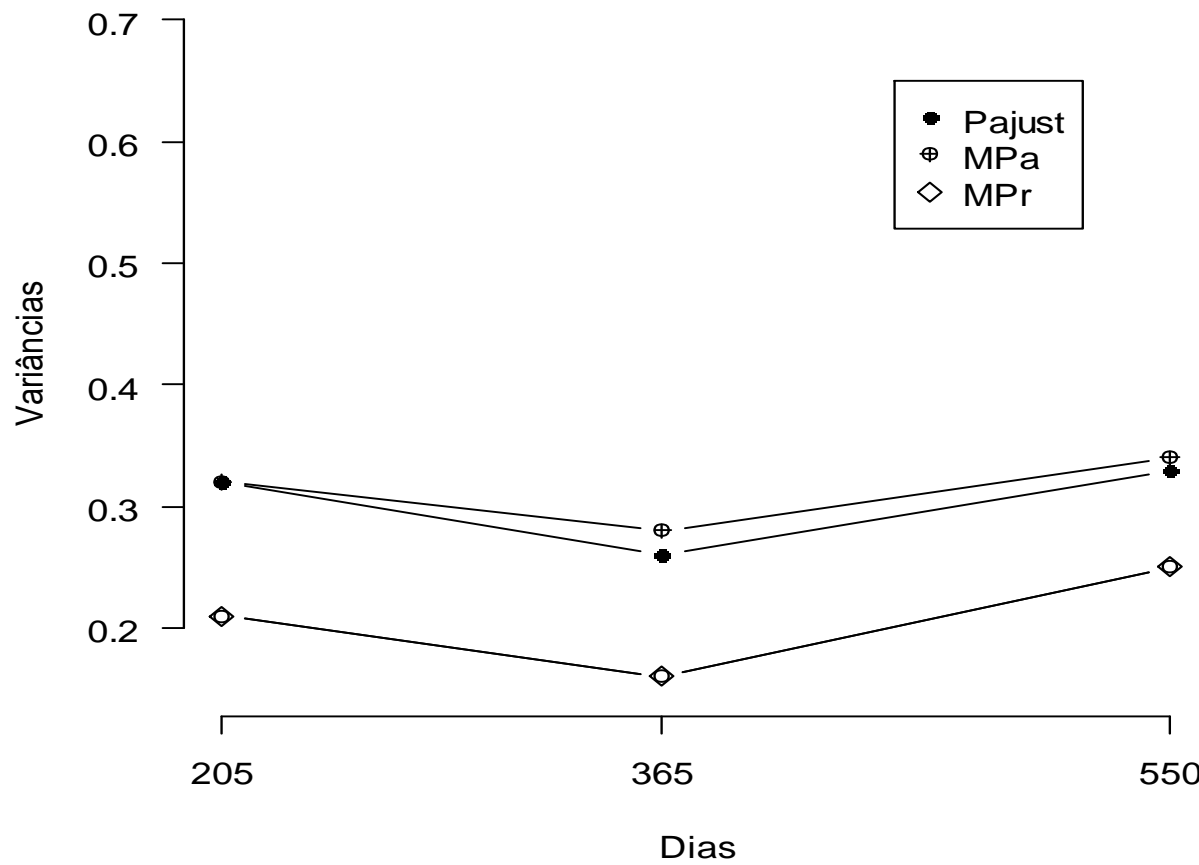

Figura 1. Relação das estimativas de variância genética aditiva e variância residual de pesos ajustados a idades-padrão 205 (PA205), 365 (PA365), 550 (PA550) dias, e respectivos pesos metabólicos ajustados a idades-padrão $205\left(\mathrm{PA}^{0,75} 205\right), 365\left(\mathrm{PA}^{0,75} 365\right), 550\left(\mathrm{PA}^{0,75} 550\right)$ dias, pesos metabólicos não ajustados a idades-padrão $205\left(\mathrm{P}^{0,75} 205\right), 365\left(\mathrm{P}^{0,75} 365\right)$ e $550\left(\mathrm{P}^{0,75} 550\right)$ dias de análises bicaracterísticas. 
As razões entre variâncias genéticas aditivas e residuais são demonstradas para cada idadepadrão. As razões calculadas a partir dos pesos metabólicos são sempre menores que aquelas dos pesos à idade-padrão. Esses resultados confirmam que os pesos metabólicos não estão sendo completamente corrigidos para os efeitos de idade, os quais estão criando variabilidade de ambiente adicional. Consequentemente, as variâncias genéticas aditivas dos pesos metabólicos têm seus valores diminuídos em relação aos pesos corrigidos, uma vez que a variância residual passa a explicar grande parte da variabilidade fenotípica. Uma forma de comparar isoladamente as reduções da variância genética aditiva e os incrementos da variância residual dos pesos metabólicos em relação aos pesos corrigidos é calcular, para cada idade, uma razão entre a variância de interesse e a média da característica. Notar-se-á que as variâncias residuais e as variâncias genéticas aditivas para o peso metabólico estão aumentadas e diminuídas, respectivamente, em relação às razões dos pesos corrigidos.
Os valores encontrados no presente trabalho (Tab. 3) indicam efetivamente que o peso metabólico não está ajustando os pesos para efeitos de idade do animal, mas sim é uma forma diferente de expressar o peso vivo do animal em pesos metabólicos. Portanto, o efeito da idade está causando o incremento da variância residual, consequentemente o efeito de idade não está sendo corrigido simplesmente por estar a elevar pesos a 0,75 .

$\mathrm{Se}$ os pesos metabólicos tivessem sido completamente corrigidos para a idade antes de serem calculados, as correlações genéticas e ambientais esperadas entre pesos corrigidos a determinada idade e pesos metabólicos corrigidos naquela idade deveriam ser próximas à unidade (Tab. 3). Da mesma forma, as correlações genéticas e de ambiente entre pesos corrigidos em diferentes idades deveriam ter valores semelhantes (mas não igual a um) quando calculadas com os pesos metabólicos não ajustados a diferentes idades.

Tabela 3. Estimativas de correlações genéticas (acima da diagonal) e correlações de ambiente (abaixo da diagonal) para características de pesos metabólicos não ajustados, pesos ajustados a idades-padrão e pesos metabólicos ajustados à idade-padrão em bovinos Brahman

\begin{tabular}{cccccccccc}
\hline & PA205 & PA365 & PA550 & $\mathrm{PA}^{0,75} 205$ & $\mathrm{PA}^{0,75} 365$ & $\mathrm{PA}^{0,75} 550$ & $\mathrm{P}^{0,75} 205$ & $\mathrm{P}^{0,75} 365$ & $\mathrm{P}^{0,75} 550$ \\
\hline PA205 & $*$ & $0,55 \pm 0,08$ & $0,72 \pm 0,06$ & $1.00 \pm 0,00$ & $0,55 \pm 0,08$ & $0,72 \pm 0,05$ & $0,76 \pm 0,04$ & $0,49 \pm 0,09$ & $0,71 \pm 0,06$ \\
PA365 & $0,43 \pm 0,02$ & $*$ & $0,92 \pm 0,03$ & $0,56 \pm 0,08$ & $1,00 \pm 0,00$ & $0,92 \pm 0,03$ & $0,57 \pm 0,08$ & $0,90 \pm 0,03$ & $0,84 \pm 0,05$ \\
PA550 & $0,39 \pm 0,02$ & $0,55 \pm 0,01$ & $*$ & $0,72 \pm 0,06$ & $0,92 \pm 0,03$ & $1,00 \pm 0,00$ & $0,68 \pm 0,07$ & $0,92 \pm 0,03$ & $0,97 \pm 0,01$ \\
$\mathrm{PA}^{0,75} 205$ & $1,00 \pm 0,00$ & $0,44 \pm 0,02$ & $0,39 \pm 0,02$ & $*$ & $0,55 \pm 0,08$ & $0,72 \pm 0,06$ & $0,76 \pm 0,04$ & $0,49 \pm 0,09$ & $0,71 \pm 0,06$ \\
$\mathrm{PA}^{0,75} 365$ & $0,43 \pm 0,02$ & $1,00 \pm 0,00$ & $0,55 \pm 0,01$ & $0,44 \pm 0,02$ & $*$ & $0,92 \pm 0,03$ & $0,56 \pm 0,08$ & $0,89 \pm 0,03$ & $0,84 \pm 0,05$ \\
$\mathrm{PA}^{0,75} 550$ & $0,39 \pm 0,02$ & $0,55 \pm 0,01$ & $1,00 \pm 0,00$ & $0,39 \pm 0,02$ & $0,55 \pm 0,01$ & $*$ & $0,68 \pm 0,07$ & $0,92 \pm 0,03$ & $0,97 \pm 0,01$ \\
$\mathrm{P}^{0,75} 205$ & $0,68 \pm 0,01$ & $0,37 \pm 0,02$ & $0,31 \pm 0,02$ & $0,68 \pm 0,01$ & $0,37 \pm 0,02$ & $0,32 \pm 0,02$ & $*$ & $0,40 \pm 0,10$ & $0,54 \pm 0,08$ \\
$\mathrm{P}^{0,75} 365$ & $0,37 \pm 0,02$ & $0,85 \pm 0,01$ & $0,51 \pm 0,01$ & $0,38 \pm 0,02$ & $0,85 \pm 0,01$ & $0,51 \pm 0,01$ & $0,27 \pm 0,01$ & $*$ & $0,79 \pm 0,05$ \\
$\mathrm{P}^{0,75} 550$ & $0,29 \pm 0,02$ & $0,40 \pm 0,01$ & $0,95 \pm 0,00$ & $0,29 \pm 0,02$ & $0,40 \pm 0,02$ & $0,95 \pm 0,00$ & $0,22 \pm 0,01$ & $0,42 \pm 0,01$ & $*$ \\
\hline
\end{tabular}

Pesos ajustados a idades-padrão 205 (PA205), 365 (PA365), 550 (PA550) dias, pesos metabólicos ajustados a idadespadrão 205 ( $\left.\mathrm{PA}^{0,75} 205\right), 365\left(\mathrm{PA}^{0,75} 365\right), 550\left(\mathrm{PA}^{0,75} 550\right)$ dias, pesos metabólicos não ajustados a idades-padrão 205 $\left(\mathrm{P}^{0,75} 205\right), 365\left(\mathrm{P}^{0,75} 365\right)$ e $550\left(\mathrm{P}^{0,75} 550\right)$ dias.

Os resultados da Tab. 3 confirmam as distorções que ocorreram nos resultados esperados das correlações quando os pesos metabólicos não foram corrigidos para idade. Por exemplo, as correlações genéticas entre PA205 com $\mathrm{P}^{0,75} 205$ e PA365 com $\mathrm{P}^{0,75} 365$ são, respectivamente, 0,76 e 0,87 , as correlações genéticas entre PA205 com $\mathrm{PA}^{0,75} 205$ e PA365 com PA ${ }^{0,75} 365$ são, respectivamente, 1,00 e 1,00. Para a correlação genética entre PA550 e $\mathrm{P}^{0,75} 550$, a correlação foi próxima da unidade, e a correlação genética entre PA550 e PA ${ }^{0,75} 550$ foi de 1,00. A correlação genética entre PA205 e PA365 foi de 0,55, enquanto aquela entre $\mathrm{P}^{0,75} 205$ e $\mathrm{P}^{0,75} 365$ foi de 0,4 , para $\mathrm{PA}^{0,75} 205$ e para $\mathrm{PA}^{0,75} 365$ foi de 0,55 . Um padrão de diminuição das correlações genéticas aditivas é observado para as outras combinações de pesos a idades-padrão (Tab. 3).

A correlação genética é uma medida do grau de associação entre os valores genéticos aditivos dos indivíduos em relação a suas características. Quando o peso metabólico não é corrigido para a idade, o efeito de ambiente passa a ter maior influência na determinação do fenótipo e dificulta a separação do componente genético 
aditivo do componente ambiental, produzindo predições de valor genético menos precisas e diminuindo as correlações genéticas entre as características de peso a idades-padrão e pesos metabólicos.

As correlações ambientais estimadas entre peso à desmama e pesos aos 12 e 18 meses de idade foram, em média, de 0,43 e 0,39. As estimativas de correlação ambiental de PA205 com PA365 e PA550 foram semelhantes às descritas por Souza et al. (2001), de 0,44 e 0,39; Malhado et al. (2002), 0,35 e 0,39, respectivamente, e inferiores às de Ferraz Filho (1996), de 0,61 e 0,51, na mesma ordem. As diferentes populações podem ter contribuído para a variação observada.

\section{CONCLUSÕES}

Conclui-se, portanto, que os pesos metabólicos não devem ser utilizados como critério de seleção pelos programas de melhoramento genético na busca de animais mais eficientes sob os pontos de vista alimentar e econômico. A utilização dessa prática pelos programas deve levar a estimativas distorcidas dos parâmetros genéticos e dos valores genéticos aditivos na população, permitindo a seleção de reprodutores que não irão contribuir para o progresso da eficiência alimentar dos rebanhos.

\section{REFERÊNCIAS}

ESTRADA-LEÓN, R.J.; MAGAÑA-MONFORTE, J.G.; SEGURA-CORREA, J.C. Estimation of genetic parameters for preweaning growth traits of Brahman cattle in Southeastern Mexico. Trop. Anim. Health Prod., v.46, p.771-776, 2014.

FERRAZ FILHO, P.B. Análise e tendência genética de pesos em bovinos da raça Nelore Mocha no Brasil. 1996. 163f. Dissertação (Mestrado) - Faculdade de Ciência Agrárias e Veterinárias de Jaboticabal, Universidade Estadual Paulista, SP.

GUIDOLIN, D.G.F.; BUZANSKAS, M.E.; RAMOS, S.B. et al. Genotype-environment interaction for postweaning traits in Nellore beef cattle. Anim. Prod. Sci., v.52, p.975-980, 2012.
HEADY, H.F. Rangeland management. [USA]: McGraw-Hill Book Company, 1975.

MALHADO, C.H.M.; SOUZA, J.C.; SILVA, L.O.C.; FERRAZ FILHO, P.B. Correlações genéticas, fenotípicas e de ambiente entre os pesos de várias idades em bovinos da raça Guzerá no estado de São Paulo. Arch. Vet. Sci., v.7, p.71-75, 2002.

MEYER, K. A program for mixed model analyses by restricted maximum likelihood: user notes. Armidale: University of New England, 2011.

PARRA-BRACAMONTE, G.M.; MARTÍNEZGONZÁLEZ, J.C.; CIENFUEGOS-RIVAS, E.G. et al. Parámetros genéticos de variables de crecimiento de ganado Brahman de registro en México Genetic parameters for growth traits. Vet. Mex., v.38, p.2, 2007.

PICO, B.A.; NESER, F.W.C.; WYK, J.V. Genetic parameters for growth traits in South African Brahman cattle. S. Afr. J. Anim. Sci., v.34, p.44-46, 2004.

PLASSE, D.; ARANGO, J.; FOSSI, H. et al. Genetic and non-genetic trends for calf weights in a Bos indicus herd upgraded to pedigree Brahman. Livest. Res. Rural Dev., v.16, p.2004, 2004.

R DEVELOPMENT CORE TEAM: a language and environment for statistical computing: $\mathrm{R}$ Foundation for Statistical Computing. Vienna, Austria: [s.n.], 2013. Disponível em: $\langle$ http://www.R-project.org $>$. Acesso em: 25 set. 2013.

SAKAGUTI, E.S.; SILVA, M.A.; QUAAS, R.L. et al. Avaliação do crescimento de bovinos jovens da raça Tabapuã, por meio de análises de funções de covariância. Rev. Bras. Zootec., v.32, p.864-874, 2003.

SOUZA, J.; SILVA, L.; MALHADO, C. et al. Estudo das correlações genéticas, fenotípicas e de ambiente entre os pesos de várias idades em bovinos da raça guzerá no estado de São Paulo. Reun. Anu. Soc. Bras. Zootec., v.38, p.609-611, 2001.

VARGAS, G.; BUZANSKAS, M.E.; GUIDOLIN, D.G.F. et al. Genetic parameter estimation for pre- and post-weaning traits in Brahman cattle in Brazil. Trop. Anim Health Prod., v.46, p.1271-1278, 2014.

ZUIN, R.G.; BUZANSKAS, M.E.; CAETANO, S.L. et al. Genetic analysis on growth and carcass traits in Nelore cattle. Meat Sci., v.91, p.352-357, 2012. 